\title{
TOWARDS INTEGRATED MICRO-MACHINED SILICON-BASED NANOPORES FOR CHARACTERIZATION OF DNA
}

\author{
Hung Chang ${ }^{1}$, Farhad Kosari ${ }^{4}$, George Andreadakis ${ }^{4}$, George Vasmatzis ${ }^{4}$, Ed Basgall ${ }^{5}$, \\ Alexander H. King ${ }^{2}$, and Rashid Bashir ${ }^{1,3}$ (bashir@ecn.purdue.edu) \\ ${ }^{1}$ School of Electrical and Computer Engineering, ${ }^{2}$ School of Materials Engineering, \\ ${ }^{3}$ Department of Biomedical Engineering, Purdue University, West Lafayette, IN 47907-1285. \\ ${ }^{4}$ Mayo Clinic, Rochester, MN 55905. \\ ${ }^{5}$ Penn State University, University Park, \\ PA 16802.
}

\section{ABSTRACT}

The direct characterization, detection, and identification of single nucleic acid molecules can revolutionize genomics, expression analysis, and many areas of biological sciences. A nanopore fabricated using micro and nano-fabrication technique is a viable sensor for such single molecule studies. Here we report the fabrication and processes characterization of silicon-based nanopores using e-beam lithography and TEMbased processing. We performed investigations on the fabrication process in depth. Experiments of metal deposition on the nanopore were also performed to observe the interactions between the metal and the surface oxide.

\section{INTRODUCTION}

The concept of characterizing biological single molecules such as nucleic acids with a single pore has been investigated in the recent years. The idea is essentially a coulter-counter based approach, in which the electric detection is achieved by fluctuations of the ionic current in the system when the interested species passes through the single pore Chang et al. [1] fabricated a silicon-based micropore and performed characterization of bacteria as they moved through the pore under an electric field. In his work, ionic currents dropped when the bacteria were passing through the micropore and the current recovered to the original level when the bacteria passed through the micropore. Characteristics of the bacteria could be extracted from the time-sampled current plots. To apply this idea to DNA and related nucleic acids, a pore with 2-4 $\mathrm{nm}$ in diameter has to be realized. Kasianowicz et al. [2] pioneered the use of a single $2 \mathrm{~nm}$ diameter $\alpha$-hemolysin channel for the electrical detection of a single DNA molecule, as the molecule traversed the channel. Meller et al. [3] later applied the same channel to discriminate between purines and pyramidines. The channel used in the above works was protein-based. A siliconbased nanopore has also subsequently been reported $[4,5]$. In Li's work [4], a feedback controlled ion milling process was used to sculpt a nanopore. A 60 $\mathrm{nm}$ hole shrank into a $1.8 \mathrm{~nm}$ nanopore after being focused by an ion beam. The same group also performed DNA trans-

Travel support has been generously provided by the Transducers Research Foundation and by the DARPA MEMS and DARPA BioFlips programs. location measurements through the nanopore [6]. Translocation times and folding of double-stranded DNA with different lengths were thus obtained. Storm et al. recently reported the fabricated of a nanopore with e-beam lithography and TEM techniques. Their method realized an in-situ observation of pore size, and the size could precisely be controlled at the nanometer level. Our fabrication process, though developed independently, is similar to [4]. In this paper, we present further details and characterization of the fabrication process for the nanopore. We also investigated placing thin metal films around the pore and explored the shrinkage of the pore with metal layers around them. The eventual goal of those preliminary works is to fabricate robust nanopores for the characterization of nucleic acid molecules within integrated biochips.

\section{NANOPORE FABRICATION}

The simplified cross sections of the process flow are illustrated in Fig. 1. The nanopore fabrication commenced with double polished SOI wafers. The buried oxide and SOI layers were 400 $\mathrm{nm}$ and $190 \mathrm{~nm}$ thick, respectively. A $100 \mathrm{~nm}$ thermal oxide was grown on the SOI wafer. An LPCVD nitride layer was deposited and etch windows $(\sim 670 \mu \mathrm{m}$ by $670 \mu \mathrm{m}$ area $)$ were formed on the backside. The nitride and oxide was etched and the handle layer of the wafer was etched from the back side in TMAH at 90C. The handle silicon layer was etched through, and the etch process stopped on the buried oxide to form a diaphragm of around $80 \mu \mathrm{m}$ by $80 \mu \mathrm{m}$ area. The nitride was stripped off in boiling phosphoric acid at $180 \mathrm{C}$, and electron beam lithography was performed on the front to define $100 \mathrm{~nm}$ by $100 \mathrm{~nm}$ openings on the diaphragm. Next, a short TMAH etch at $60 \mathrm{C}$ was performed to etch the SOI, in an inverted pyramid shape, down to the buried oxide. When the buried oxide was removed, a pore of less than $100 \mathrm{~nm}$ diameter was formed. Thermal oxide was then grown to shrink the pore to less than $50 \mathrm{~nm}$. Finally, the pore was examined and processed in a transmission electron micrograph and shrank to the desired nanometer dimension utilizing the in-situ observation.

\section{RESULTS AND DISCUSSION}

The metamorphosis of the pore by TEM is a complicated process. Not only shrinkage but also expansion of the pore occurred in the experiments. 
Fig. 2 shows TEM images of pore shrinking in the TEM. A $91 \mathrm{~nm}$ by $94 \mathrm{~nm}$ pore (before the final oxidation) was used to form a $75 \mathrm{~nm}$ by $78 \mathrm{~nm}$ pore after $100 \mathrm{~nm}$ thermal oxide was grown on the pore (Note: TEM pictures are shown when pore was 51 $\mathrm{nm}$ by $54 \mathrm{~nm}$ ). Subsequent observation in the TEM resulted in shrinking of the pore, as shown in Figure 2. The average shrinkage rate of one of the pore was $0.57 \mathrm{~nm} / \mathrm{min}$, as shown in Fig. 3.

The pore shown in Fig. 4 was $168 \mathrm{~nm}$ by 172 $\mathrm{nm}$ after a $90 \mathrm{~nm}$ thermal oxide was grown on the pore. The pore expanded to $206 \mathrm{~nm}$ and $208 \mathrm{~nm}$ within $58 \mathrm{~min}$. Occurrences of shrinkage or expanding depend on the initial pore size and the thickness of the oxide grown on the pore. The critical radius of the pore was suggested in [5] and [7] as: $r_{c}=d / 2$, where $r_{c}$ is the critical radius, $d$ is the oxide thickness. The pore shrinks if the radius is smaller than $d / 2$; it expands, if radius is greater than $\mathrm{d} / 2$. When being examined under high electron energy, the oxide covering the pore flows to reduce the surface energy. A void region, the pore, has lower surface energy when the radius of the pore is below the critical value. In the opposite case, the void region has a higher surface energy; the pore expands. Table 1 shows radii, oxide thicknesses and shrinkage/expanding of some of our pores. The table shows that indeed the pores shrink or expand depending on the ratio of the pore radius to the oxide thickness, and validates the proposed model i.e. $\mathrm{r} / \mathrm{d}>0.5$ results in expanding the pore and $\mathrm{r} / \mathrm{d}<0.5$ results in shrinking the pore.

The shrinkage rate varied over time and especially as a function of starting size. The pore seemed to shrink faster at the beginning, and the initial shrinkage rates were between 0.6 to 2.6 $\mathrm{nm} / \mathrm{min}$. The rates were also observed to be related to the oxide thickness; the pore with a thicker oxide had a higher initial shrinkage rate. The shrinkage rate reduced down to $0.3 \mathrm{~nm} / \mathrm{min}$ in all cases, when the pore radius went below $10 \mathrm{~nm}$. Table 2 shows the initial shrinkage rates and the oxide thicknesses of some pores. Figure 3 shows the change shrinking rate vs. time for two pore diameters. The average shrinkage rates are the slopes of the fitted curves, and it is obvious that pores with different oxide thicknesses have different shrinkage rates.

It was also found that non-circular pores could be rounded by the TEM processing. Fig. 5 shows TEM images of an oval pore turning into a rounded one. Fig. 6 shows the plot of the dimensions of the pore (the long and the short axes of the oval pore) vs. time. The shrinkage rates of the long and the short axes were obviously different. The short axis didn't change much for sometime while when the long axis was shrinking. Once the axes became the same size (a circle), then the newly formed circular pore continues to shrink further. This interesting fact is also related to a surface energy of the pore. According to the observation described in the last paragraph, the pore initially shrank faster and shrinkage slowed down after a while. This phenomenon gives a hint that a larger vacant space (as long as below the critical radius) has a larger surface energy comparing to a smaller one. Therefore, the long axis has a higher shrinkage rate than the short one in an oval pore. It can be postulated that the surface energy of the pore due to the long axis is locally higher than that due to the short one, and hence the oxide along the long axis flows faster.

Preliminary experiments involving deposition of a thin metal layer on the pore was also performed before the shrinkage step in TEM. The electron beam was then focused on the pore covered with both the surface oxide and the thin metal layer. This experiment was to investigate if a thin metal layer moves under e-beam irradiation, along with the pores. Two sets of chips were prepared, one with 10 $\mathrm{nm}$ Ti only, and one with $10 \mathrm{~nm}$ Ti and $5 \mathrm{~nm}$ gold film. Fig. 7 shows the case with the $10 \mathrm{~nm}$ Ti film. In this case, the metal seems to move along with the pore as it shrinks, since the metal edge cannot be clearly discerned. Fig. 8 shows pore with the $10 \mathrm{~nm}$ $\mathrm{Ti}$ and the $5 \mathrm{~nm}$ gold. In this case, the film does not seem to move along with the pore as the metal edge can be clearly seen away from the pore itself. More experiments need to be performed and this information can be very useful when designing the placement of metal electrodes at the nanopore for tunnel electrodes to laterally measure the passage of DNA through the pore.

\section{CONCLUSION}

We have fabricated nanopores in silicon-based membranes using micro and nano-fabricated processes along with Transmission Electron Microscope (TEM)-induced pore size reduction. The process is shown to produce nano-pores, even when starting with non-circular openings. Thin metal films could also be placed on the pore and were found to shrink and move along with the oxide for some films. The measurement of DNA translocation through these nanopores is in progress.

\section{ACKNOWLEDGMENTS}

The authors would like to acknowledge the support of NIH NIBIB (R21 RR15118-01) and NASA Institute of Nanoelectronics and Computing at Purdue (Award no. NCC 2-1363) for funding the work, and the Birck Nanotechnology Center for fabrication facilities at Purdue University.

\section{REFERENCES}

1. H. Chang, A. Ikram, T. Geng, F. Kosari, G. Vasmatzis, A. Bhunia, and R. Bashir, "Electrical characterization of microorganisms using microfabricated devices", Journal of Vacuum Society and Technology B, 20, 2058 (2002).

2. John. J. Kasianowicz, Eric Brandin, Daniel Branton and David W. Deamer, "Characterization of individual polynucleotide molecules using a membrane channel", Proc. Natl. Acad. Sci., 93, 13770 (1996). 
3. Amit Meller and Daniel Branton, "Single molecule measurements of DNA transport through a nanopore", Electrophoresis, 23, 2583 (2002).

4. Jiali Li, Derek Stein, Ciaran McMullan, Daniel Branton, Michael J. Aziz and Jene A. Golovchenko, "Ion-beam sculpting at nanometer length scales" Nature, 412, 166 (2001)

5. A. J. Storm, J.H. Chen, X.S. Ling, H.W. Zanderbergen and C. Dekker, "Fabrication of solidstate nanopores with single-nanometre precision", Nature Materials, 2, 537 (2003).

6. Jiali Li, Marc Gershow, Derek Stein, Eric Brandin and Jene A. Golovchenko, "DNA molecules and configurations in a solidstate nanopore microscope" Nature Materials, 2, 611 (2003).

7. M. Lanxner, C. L. Bauer and R. Scholtz, "Evolution of hole size and shape in $\{100\},\{110\}$ and $\{111\}$ monocrystalline thin film of gold", Thin Solid Film, 150, 323 (1987).

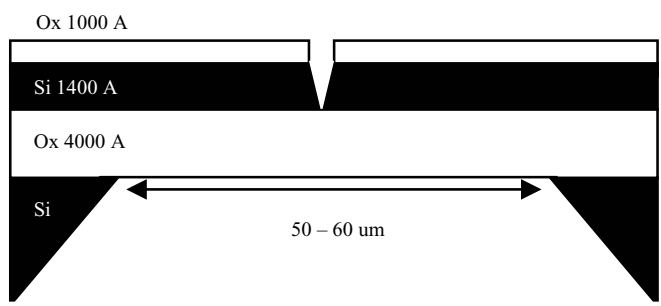

(a) After TMAH etch on both sides.

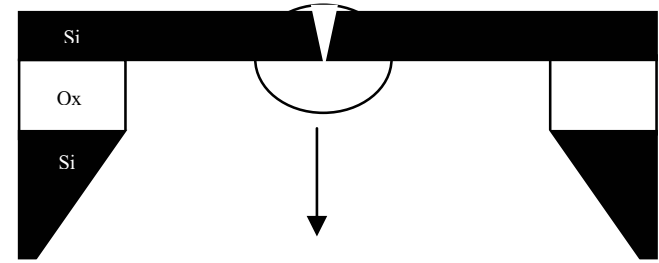

(b) A pore was opened after buried oxide layer was removed.
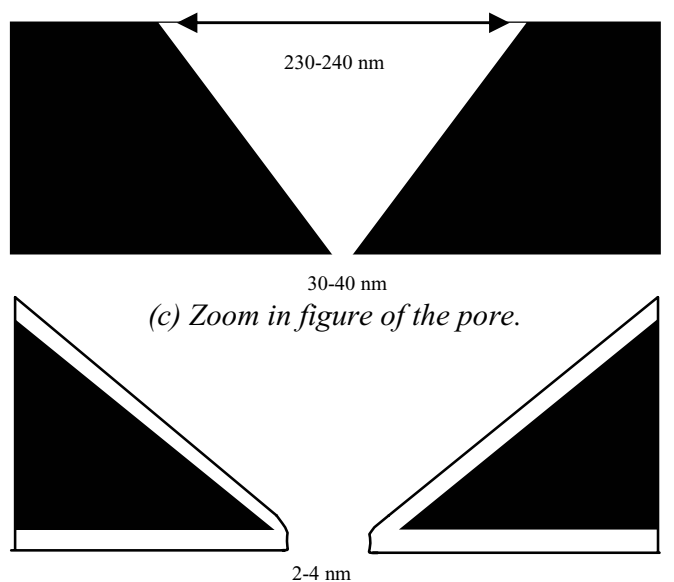

(d) Grow thermal oxide and shrink the pore by TEM.

Figure 1. The simplified cross sections of the process flow.

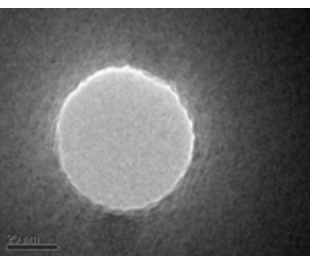

(a) $51 \mathrm{~nm} \times 53 \mathrm{~nm}$ pore after the last thermal oxidation

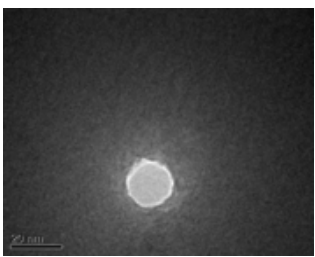

(c) $17 \mathrm{~nm} \times 17 \mathrm{~nm}$ 45 mins in TEM

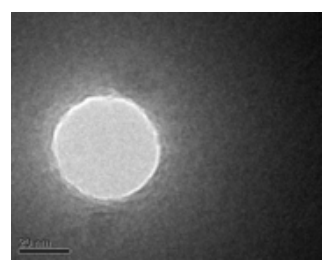

(b) $38 \mathrm{~nm} \times 40 \mathrm{~nm}$ 20 mins in TEM

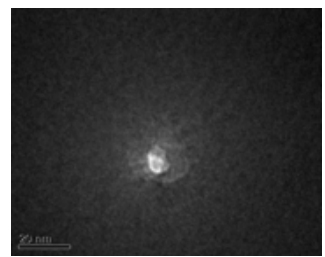

(d) $4.2 \mathrm{~nm} \times 4.6 \mathrm{~nm}$ 83 mins in TEM

Figure $2(\boldsymbol{a}-\boldsymbol{d})$. Shrinking of the nano-pore to the desired size using TEM. Fabrication was in situ observed and pore size could be accurately controlled at nanometer level. All images are in 1,000,000 X.

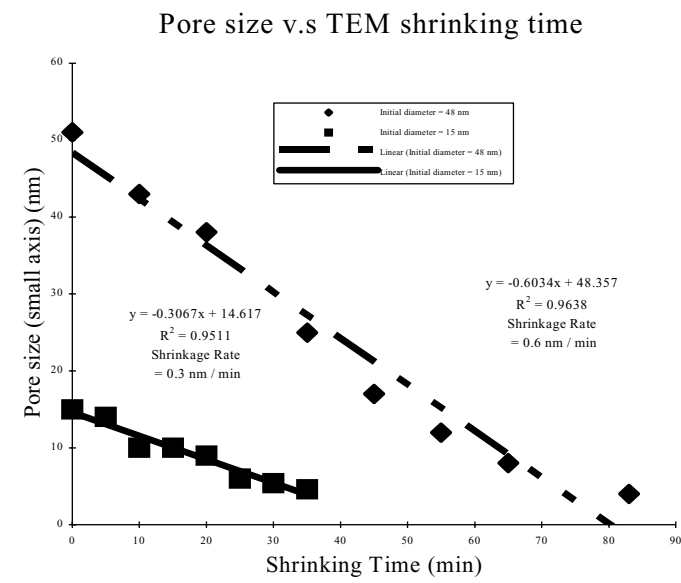

Figure 3. Slopes in the plot are the shrinkage rates. Different initial pore size had different shrinkage rates.

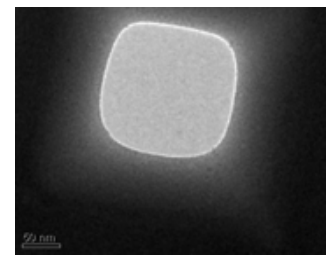

(a) $168 \mathrm{~nm} \times 172 \mathrm{~nm}$ Pore after the last Thermal oxidation

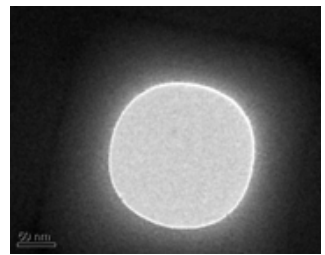

(b) $187 \mathrm{~nm} \times 189 \mathrm{~nm}$ after 20 mins in TEM 


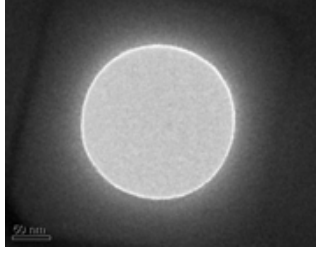

(c) $200 \mathrm{~nm} \times 201 \mathrm{~nm}$ 45 mins in TEM

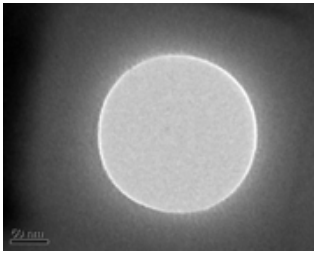

(d) $206 \mathrm{~nm} \times 208 \mathrm{~nm}$ 58 mins in TEM
Figure $4(\boldsymbol{a}-\boldsymbol{d})$. Pore with larger initial size (just prior in TEM) expanded, not shrank. All images are in $300,000 X$.

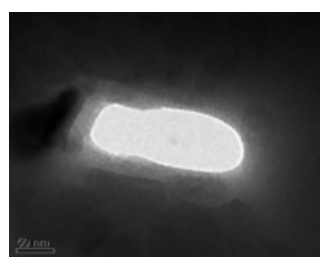

(a) $69 \mathrm{~nm} \times 198 \mathrm{~nm}$ Initial pore

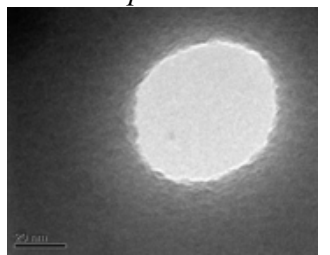

(c) $48 \mathrm{~nm} \times 57 \mathrm{~nm}$ 250 mins

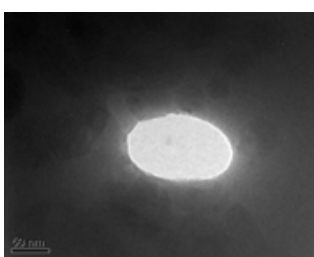

(b) $80 \mathrm{~nm} \times 156 \mathrm{~nm}$ 90 mins

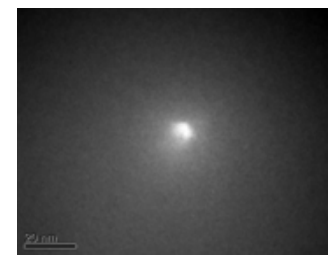

(d) $3.3 \mathrm{~nm} \times 3.5 \mathrm{~nm}$ 334 mins
Figure 5. Oval pore turned round by TEM. The first two images are in $300,000 \mathrm{X}$, and the last two images are in 1,000,000 X.

Short axis shrinkage v.s. long axis shrinkage in an oval pore

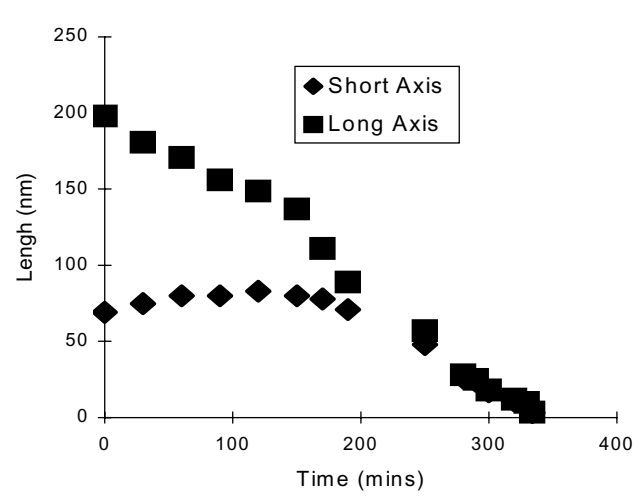

Figure 6. Long and short axes had different shrinkage rates. Diamond marks are the trends of the short axis and square ones are for the long axis.
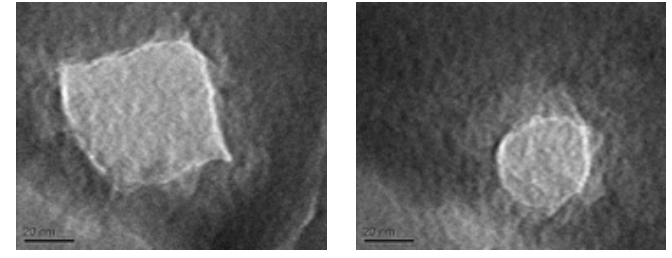

Figure 7. Nanopore with $10 \mathrm{~nm}$ Ti deposition. (a) Initial image of the pore. (b) Pore with $10 \mathrm{~nm} \mathrm{Ti}$ after 30 mins TEM observation.
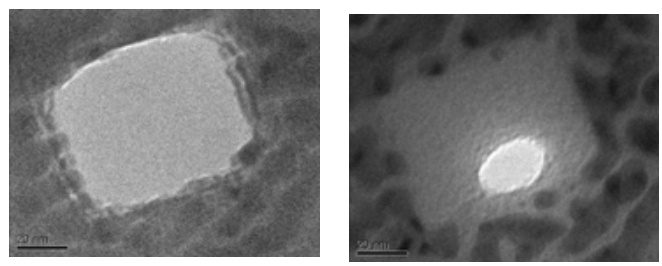

Figure 8. Nanopore with $10 \mathrm{~nm}$ Ti and $5 \mathrm{~nm}$ gold deposition. (a) Initial image of the pore. (b) Pore shrank after 30 mins.

\begin{tabular}{|c|c|c|c|}
\hline $\begin{array}{c}\text { Pore Name } \\
\text { Exp./Shr. }\end{array}$ & $\begin{array}{c}\text { Average } \\
\text { radius of } \\
\text { the pore } \\
\mathrm{r}(\mathrm{nm})\end{array}$ & $\begin{array}{c}\text { Grown } \\
\text { oxide } \\
\text { thickness } \\
\mathrm{d}(\mathrm{nm})\end{array}$ & $\begin{array}{c}\text { The ratio } \\
\text { of r/d }\end{array}$ \\
\hline $\begin{array}{c}103003 \_4 \\
\text { Shrunk }\end{array}$ & 9 & 79 & 0.11 \\
\hline $\begin{array}{c}102503 \_4 \\
\text { Shrunk }\end{array}$ & 38 & 100 & 0.38 \\
\hline $\begin{array}{c}110603 \_2 \\
\text { Shrunk }\end{array}$ & 51.5 & 130 & 0.40 \\
\hline $\begin{array}{c}\text { 091603_6m } \\
\text { Expanded }\end{array}$ & 82 & 90 & 0.91 \\
\hline $\begin{array}{c}082703 \_5 \mathrm{~m} \\
\text { Expanded }\end{array}$ & 138 & 120 & 1.15 \\
\hline $\begin{array}{c}\text { 091603_7m } \\
\text { Expanded }\end{array}$ & 110 & 54 & 2.04 \\
\hline
\end{tabular}

Table 1. Radii, oxide thicknesses and shrinkagelexpanding of some of our pores. For ratio of $r / d>0.5$, the pores generally expanded and for ratio $r / d<0.5$, the pores generally shrank.

\begin{tabular}{|c|c|c|c|}
\hline $\begin{array}{c}\text { Pore } \\
\text { Name }\end{array}$ & $\begin{array}{c}\text { Grown } \\
\text { oxide } \\
\text { thickness } \\
\mathrm{d}(\mathrm{nm})\end{array}$ & $\begin{array}{c}\text { Initial } \\
\text { pore } \\
\text { radius } \\
\mathrm{r}(\mathrm{nm})\end{array}$ & $\begin{array}{c}\text { Initial } \\
\text { shrinkage } \\
\text { rate } \\
(\mathrm{nm} / \mathrm{min})\end{array}$ \\
\hline $103003 \_4$ & 79 & 9 & 0.6 \\
\hline $102503 \_4$ & 100 & 38 & 1.6 \\
\hline $110603 \_2$ & 130 & 51.5 & 2.6 \\
\hline
\end{tabular}

Table 2. The last grown oxide, initial pore radius and the initial shrinkage rates of some pores. 